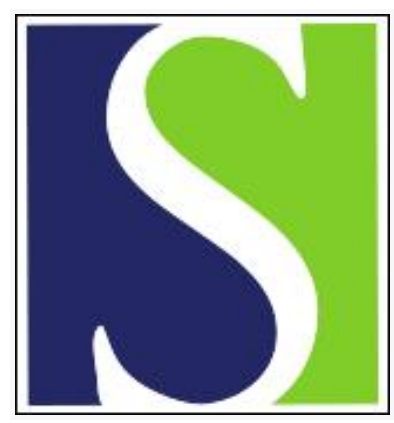

Scand J Work Environ Health 2006;32(4):285-293

https://doi.org/10.5271/sjweh.1012

Issue date: 31 Aug 2006

Work organization and musculoskeletal injuries among a cohort of health care workers

by Koehoorn M, Demers PA, Hertzman C, Village J, Kennedy SM

Affiliation: Department of Health Care \& Epidemiology, University of British Columbia, 5804 Fairview Avenue, Vancouver, British Columbia, V6KT 1Z3, Canada. mieke.koehoorn@ubc.ca

Refers to the following texts of the Journal: 1998;24(1):54-61

1995;21(6):427-434

The following article refers to this text: 2014;40(4):400-410

Key terms: back injury; cohort study; epidemiology; health care worker; health personnel; musculoskeletal injury; occupational health; work organization

This article in PubMed: www.ncbi.nlm.nih.gov/pubmed/16932826

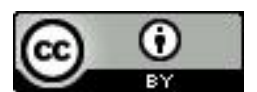




\title{
Work organization and musculoskeletal injuries among a cohort of health care workers
}

\author{
by Mieke Koehoorn, PhD, 1, 2, 3 Paul A Demers, PhD, ${ }^{1,2}$ Clyde Hertzman, PhD, ${ }^{2}$ Judy Village, MSc, ${ }^{1}$ Susan \\ M Kennedy, PhD', 2
}

\begin{abstract}
Koehoorn M, Demers PA, Hertzman C, Village J, Kennedy SM. Work organization and musculoskeletal injuries among a cohort of health care workers. Scand J Work Environ Health 2006;32(4):285-293.
\end{abstract}

\begin{abstract}
Objectives This study investigated the relationship between work-organization factors (job control, job demands, and workload measures) and the risk of lower-body musculoskeletal injury among health care workers.

Methods A four-year, retrospective cohort study of 3769 health care workers was carried out in one acute care hospital in the Canadian province of British Columbia. A job-exposure matrix was constructed for the workorganization factors from survey and administrative data and assigned to workers on the basis of their occupation and department of employment. Musculoskeletal injuries resulting in workers' compensation claims were ascertained from the injury database of the hospital's Occupational Health and Safety Department.

Results In the final Poisson models adjusted for demographic and biomechanical factors, an increased risk for compensated musculoskeletal injuries of the lower back and lower limb was related to low job control [relative risk (RR) 1.64, 95\% confidence interval (95\% CI) 1.08-2.49] and workload defined by working during periods of high absenteeism within a department (RR 2.10, 95\% CI 1.61-2.98). The risk also increased with more biomechanical demands in an occupation and with a recent previous injury.

Conclusions The results indicate that work-organization characteristics (job control and workload) were associated with an increased risk of musculoskeletal injuries resulting in a compensation claim. These associations remained after the effect of demographic and biomechanical factors was taken into consideration. The association with workload measured by departmental levels of absenteeism should be explored further in future studies as reverse causality (musculoskeletal symptoms resulting in absenteeism) could not be fully ruled out in the current study.
\end{abstract}

Key terms back injury; cohort study; epidemiology; health personnel; occupational health.

Reports using data from workers' compensation claims indicate that health care workers are at greater risk of work-related injuries than other workers and that musculoskeletal injuries are the major source of work-related disability in the Canadian health care sector. In the province of British Columbia, for example, health care workers incurred 4 claims per 100 worker-years in 2003 as opposed to 3 claims per 100 worker-years for all workers (1).

The prevalence of work-related musculoskeletal injuries among health care workers and the associated economic impact imply a substantial public health burden. Researchers have directed their attention to understanding the risk factors for musculoskeletal injuries to re- duce the magnitude and severity of the problem. Of particular interest is the growing body of evidence indicating that adverse work-organization characteristics are among the multiple risk factors thought to be etiological in nature $(2,3)$. Work-organization characteristics represent aspects of the more global work environment, such as supervisory support, control over tasks, and workload levels. The job-strain model developed by Karasek $(4,5)$ identifies two important elements of work organization that have been linked to several health outcomes among workers $(6,7)$, namely, psychological job demands and decision latitude or control over worktasks. A systematic review of cohort and case-control studies identified evidence for an association between

1 School of Occupational and Environmental Hygiene, University of British Columbia, Vancouver, Canada.

2 Department of Health Care and Epidemiology, University of British Columbia, Vancouver, Canada.

3 Institute for Work and Health, Toronto, Ontario, Canada.

Reprint requests to: Dr M Koehoorn, Department of Health Care \& Epidemiology, University of British Columbia, 5804 Fairview Avenue, Vancouver, British Columbia, V6KT 1Z3, Canada. [E-mail: mieke.koehoorn@ubc.ca] 
back pain and low social support, low job satisfaction, and low decision latitude in the workplace (3).

Several studies have examined more-objective measures of workload levels on musculoskeletal outcomes. The definitions of workload varied from overtime among transit workers (8) to the percentage of productivity norms for sewing machine operators (9) to organizational downsizing among municipal workers (10). It is not surprising, given this diversity, that results are inconclusive. However, Kivimäki and his colleagues (10) identified an almost threefold increased odds of musculoskeletal pain and an almost sixfold increased odds of musculoskeletal sickness absence among municipal workers in association with major periods of downsizing. Periods of major downsizing were characterized by increased levels of physical demands. Furthermore, a positive association was found for workload measured by patient-nurse ratios and musculoskeletal injuries among health care workers (11). Workload levels have been consistently identified in North America as a main determinant of work environment conditions for health care workers. Focused discussions with health care workers as part of the development of this study identified periods of overtime and periods working when experienced co-workers are absent from work, even when replaced by temporary workers, as having the highest workload levels. The analysis of workload measures is considered exploratory given the lack of validated measures and equivocal findings in the literature.

Few studies of musculoskeletal etiology have investigated the role of work-organization factors or studied them simultaneously with biomechanical factors in the health care sector. Only two longitudinal studies were found in the published literature. In a 3-year follow-up of 285 Swedish nursing personnel (12), a higher level of job strain (combination of low job control and high psychological demands) was associated with an increased risk of self-reported musculoskeletal symptoms. The risk was higher when combined with high physical exertion on the job. In a 10-year prospective study of 210 French nurses (13), the presence of two or more work-related stress factors (ie, shortage of staff, mental load, relationships with colleagues) was associated with an elevated risk of self-reported musculoskeletal pain, although only the risk for neck pain was statistically significant. Both studies were based on small sample sizes, included only nursing personnel, and relied on selfreported estimates of biomechanical and work-organization exposures and musculoskeletal outcomes.

With sufficient sample size to support the statistical analyses, our study was designed to include both nursing and nonnursing personnel, as well as independently measured biomechanical factors. A job-exposure matrix was developed from survey and administrative data for independent indicators of work organization. The pur- pose of our study was to investigate the effect of workorganization factors (job control, job demands, and departmental workload levels) and biomechanical factors on the risk of compensated musculoskeletal injuries among health care workers using a multifactor analytic model that also included demographic factors.

\section{Study population and methods}

\section{Study design and study sample}

This study employed a retrospective cohort design to investigate the individual-level risk of musculoskeletal injury in association with work organization, biomechanical factors, and demographic factors. Workers in the cohort were enumerated from the personnel records of an acute-care hospital in Vancouver, British Columbia, and included nursing personnel (eg, registered nurses, nursing aides), support staff (eg, housekeeping, dietary, and clerical workers), other health care professionals (eg, pharmacists and therapists), and administrative staff employed by the hospital for a minimum of 1 month between January 1992 and December 1995 inclusive. The follow-up period was defined by the availability of data. Physicians and students were ineligible due to a lack of outcome and exposure data.

\section{Data sources and measures}

Personnel records available from the hospital's human resources department were used to enumerate the study sample. These records included the worker's occupational history (position and department) at the hospital and were used to construct exposure records for each month of the follow-up period. Data on gender, age, and years of experience were also available from the employment records. Age was divided into quartiles for the analyses (<30 years, 30-39, 40-49, $\geq 50$ years) as was years of experience in an occupation $(<2$ years, $2-5,6-$ $10,>10$ years).

The outcome of interest was lower-body musculoskeletal injuries resulting in a workers' compensation claim obtained from a database maintained by the hospital's Occupational Health and Safety (OHS) Department. Hospital procedures require workers to submit a written report to the OHS Department describing incidents at work that affect their physical health. Reports with injury dates from January 1992 to December 1995 inclusive $(\mathrm{N}=3836)$ were read by the principal investigator and coded for strains or sprains involving the musculoskeletal system. A report was coded as a lower-body injury if it involved the lumbar back or lower limb. The coder was blinded to the demographic and occupational background of the worker who filed the report. 
Injuries caused by a sudden impact of external force such as falls from heights or being struck by objects were excluded, as were fractures and lacerations. Reports were read and coded on two separate occasions, and coding discrepancies were resolved by a second coder. A field for workers' compensation benefits was used to identify those musculoskeletal injuries that resulted in an accepted time-loss claim. Individual employment data were linked to a worker's injury report by a unique but anonymous study identifier.

A work-organization exposure matrix for measures of job control and job demands was developed from individual responses to Karasek's Job Content Questionnaire (JCQ) (4). The questionnaire was administered to three random samples of workers at the hospital in 1991 $(\mathrm{N}=250), 1994$ ( $\mathrm{N}=313)$, and 1995 ( $\mathrm{N}=298)$. The subscale for psychological job demands is the sum of five items measuring whether the job is mentally strenuous. The job control scale is the sum of two subscales measuring skill discretion and decision authority. The psychometric properties of the Job Content Questionnaire have been reported elsewhere (14). On the basis of a similar matrix by Johnson \& Stewart (15) and with the use of linear regression in SAS (16), the job control and job demand scores were analyzed from the 861 survey respondents and models built to predict scores based on significant individual and occupational variables. According to these predictive models, workers in the study sample were linked with their specific job control and demand scores on the basis of their characteristics. Individual exposure scores were updated over the study period as a worker changed from one occupation or unit of employment to another.

Workload measures were constructed from monthly, department-level data on absenteeism and overtime hours maintained by the hospital's Human Resources Department. Absenteeism and overtime were calculated as the proportion of total paid hours within each department of the hospital $(\mathrm{N}=138)$ by month. These proportions were assigned to the workers in the study sample by their department of employment and month of follow-up (department-level measures assigned to workers). Absenteeism hours due to workers' compensation disability were excluded from the workload measure. The measures of job control, absenteeism, and overtime were divided into the following quartiles based on the distribution of the variable in the total study sample: a reference category or the lowest exposure group (ie, those with high job control or a low proportion of departmental absenteeism or overtime), a medium-low exposure group, a medium-high exposure group, and a high exposure group. Because of the little variation in the distribution of scores, job demands were categorized into tertiles with the median score as the medium exposure category and scores above and below the median assigned to the high and low exposure categories, respectively.

Biomechanical factors were assessed at the occupation level. A total of 183 direct observations were completed for a minimum of two workers (range 2-12) in each hospital occupation (and department specific) during a regular or usual shift. The principal investigator completed all of the assessments over a 12-month period under the supervision and training of a certified ergonomist in the health care industry. Occupations were department specific (eg, nurse in emergency department, nurse in pediatrics, dietary aide in diabetes clinic, dietary aide in kitchen). Checklists developed by the United States Occupational Safety and Health Administration (OSHA) (17) were used to quantify biomechanical factors based on the observed presence, frequency, duration, and magnitude of 16 lower-body factors related to awkward postures, contact stress, vibration, pushing or pulling, manual handling, and patient lifting. Biomechanical scores were assigned to workers in the cohort on the basis of their occupation and department of employment and updated over the 4-year study period as workers changed jobs. The OSHA instrument was designed in such a way that exposure to at least two risk factors for more than half of a workshift would result in a score higher than 5 and would indicate a risk of injury to the worker in association with biomechanical demands. The sum of scores for the biomechanical factors was categorized as low-risk for scores of 2 or less, medium for 3-5, high for 6-8, and very high for scores of 9 or higher.

\section{Data analysis}

Poisson regression was used to analyze the individual risk of compensated musculoskeletal injuries per person-month of follow-up by work-organization and biomechanical factors, adjusted for demographic factors using EGRET (18). Demographic variables were entered first into the model, followed by the biomechanical variables, and then the job-control and job-demands variables. As an exploratory analysis, the workload measures based on department-level overtime, and absenteeism hours were entered next in the models to estimate their effect on other variables. The workload variables for overtime and absenteeism were entered into separate models as two alternate measures of workload.

Multiple musculoskeletal injuries were recorded for some study participants over the study period. In such circumstances, within-person correlation may bias the estimates of the risk ratios (19). Time since previous injury was divided into quintiles to account for multiple events per worker ( $<3$ months, 3-6, 7-12, 13-24, and $>24$ months or no prior injury). This variable was added last to the models to estimate its effect on the other 
variables. To investigate the effect of including multiple injuries per worker, analyses were re-run for (i) first injury only, censoring all subsequent injuries and ignoring the variable "time since previous injury", (ii) first event only between 1993 and 1995, including a variable for a prior injury in 1992, and (iii) multiple injuries between 1993 to 1995 for workers who were injury free in 1992, including the variable for time since previous injury.

The study protocol was approved by the University of British Columbia's Research and Ethics Committee, the hospital's Research and Ethics Committee, and the hospital's Freedom of Information and Protection of Privacy Officer.

\section{Results}

\section{Study sample}

Altogether 4146 workers were employed by the hospital between January 1992 and December 1995, of whom 3769 were included in the analyses (table 1). Of these, 254 workers were excluded for incomplete demographic and occupational data, and 123 for employment of less than 1 month. The 377 excluded workers did not differ significantly from the study sample by demographic or occupational characteristics. The study sample contributed 126877 person-months of observation during the period of follow-up.

\section{Musculoskeletal injuries}

During the study period, 3836 injuries were reported to the occupational health and safety department of the hospital. A total of $33 \%(\mathrm{~N}=1262)$ involved a musculoskeletal sprain or strain, of which 1166 were included in the analysis for eligible workers. Over half of the strains and sprains $(\mathrm{N}=675)$ resulted in a worker's compensation claim, of which 454 involved the lower body
$(67.3 \%)$. The overall rate of lower-body injuries resulting in a compensation claim was 4.1 injuries per 100 person-years. Approximately $26 \%$ of the injured workers had more than one compensation claim during the study period.

\section{Biomechanical scores}

The mean scores for biomechanical demands among the health care workers was 4.6 for lower-body factors (range $0-14$ ). Altogether $43 \%$ of the occupations held by the cohort members during the follow-up period had lower-body biomechanical scores greater than 5, signaling occupations at risk for musculoskeletal problems due to biomechanical factors (table 2). In the analysis of variance, the mean biomechanical scores were statistically different across age, gender, experience, and occupation categories. Younger workers and men had somewhat higher scores than older workers and women. Security, housekeeping, and care aide workers had the highest mean scores for biomechanical demands.

\section{Work-organization scores}

The job-control scores increased with increasing age and work experience, while scores for job demands decreased with age and experience (table 2). The women had higher levels of work-organization factors than the men, the result indicating, on the average, more control, psychological demands, and workload pressures in their occupations. On the average, the workers in dietary, housekeeping, and physical plant occupations had the lowest levels of job control, while security officers, clerical workers, and administrators had the highest levels of psychological demands.

The amount of absenteeism that a worker was exposed to in their department during any month of follow-up was, on the average, $4.2 \%$ of the total paid hours. On the average, the amount of overtime that workers were exposed to in their department during any month

Table 1. Characteristics of the Health Care Worker Study cohort ( $N=3769), 1992-1995$.

\begin{tabular}{|c|c|c|c|c|c|c|c|}
\hline & \multirow[t]{2}{*}{$\mathrm{N}$} & \multirow[t]{2}{*}{$\%$} & \multicolumn{2}{|c|}{ Age at entry into study (years) } & \multicolumn{2}{|c|}{ Employment at hospital (years) } & \multirow[t]{2}{*}{ Women (\%) } \\
\hline & & & Mean & SD & Mean & SD & \\
\hline Cohort characteristics & 3769 & 100 & 37.1 & 9.9 & 5.0 & 6.1 & 79.0 \\
\hline \multicolumn{8}{|l|}{ Occupation } \\
\hline Patient care & 1613 & 42.8 & 37.5 & 9.5 & 5.1 & 5.9 & 86.4 \\
\hline Clerical & 545 & 14.5 & 36.2 & 11.0 & 5.2 & 6.4 & 94.1 \\
\hline Support services & 471 & 12.5 & 36.9 & 10.9 & 5.3 & 6.5 & 46.7 \\
\hline Clinical professional & 283 & 7.5 & 33.3 & 9.2 & 2.4 & 3.8 & 78.4 \\
\hline Technicians & 283 & 7.5 & 35.1 & 8.9 & 4.5 & 5.3 & 79.9 \\
\hline Management & 264 & 7.0 & 42.5 & 8.8 & 8.0 & 7.6 & 71.6 \\
\hline Patient testing & 184 & 4.9 & 34.6 & 8.1 & 4.4 & 5.4 & 77.2 \\
\hline Other & 126 & 3.3 & 40.0 & 7.8 & 4.1 & 6.9 & 62.7 \\
\hline
\end{tabular}


of follow-up was $0.8 \%$ of the total paid hours. The workers working in housekeeping and security occupations had, on the average, higher levels of absenteeism and overtime hours in their departments than those in other occupations did.

\section{Risk factors for musculoskeletal injuries}

In the final models adjusted for biomechanical and demographic characteristics, the risk for lower-body musculoskeletal injuries resulting in a claim was significantly increased for the employees working in occupations with lower levels of job control (adjusted RR 1.64 for medium-low control and a slight drop in the estimate for the lowest levels of control RR 1.58) and for those working during periods of higher workload defined by higher levels of departmental absenteeism (adjusted RR 2.19 for the highest quartile and RR 1.71 for the second highest quartile), when compared with the workers in jobs with high control and those working during periods of low absenteeism, respectively (table 3 ).

The workers from medium (adjusted RR 2.12) to very high levels (adjusted RR 3.86) of biomechanical demands had consistently higher risk for lower-body injury than those with low biomechanical demands (table 3). The effect of biomechanical demands remained the strongest independent association observed between the predictor variables and the risk for lower-body injury.

The gender difference for lower body injuries did not remain significant in the final model adjusted for biomechanical demands (adjusted RR 1.06 for the men relative to the women). The workers with 2-5 years of experience in their occupation had the higher risk of lower-body injury (adjusted RR 1.62) when compared with the workers with more than 10 years of experience, although those with the least amount of experience also had an elevated risk of injury (adjusted RR 1.44). Compared with the workers with no prior musculoskeletal injury, those injured within the last 3 months were almost four times as likely to have another lower-body injury resulting in a claim.

The results of the additional analyses investigating risks associated with the first claim only, one event only, or claim-free status for first year of follow-up did not change the conclusions from the multivariable models (results not shown).

\section{Discussion}

Our results indicate that work-organization characteristics were associated with an increased risk of musculoskeletal injuries resulting in a compensation claim. These associations remained after the effect of demo-
Table 2. Scores for biomechanical demands and predicted work organization variables for the cohort of health care workers ( $N=3769)$ - 1 January 1992-31 December 1995. (RN = registered nurse)

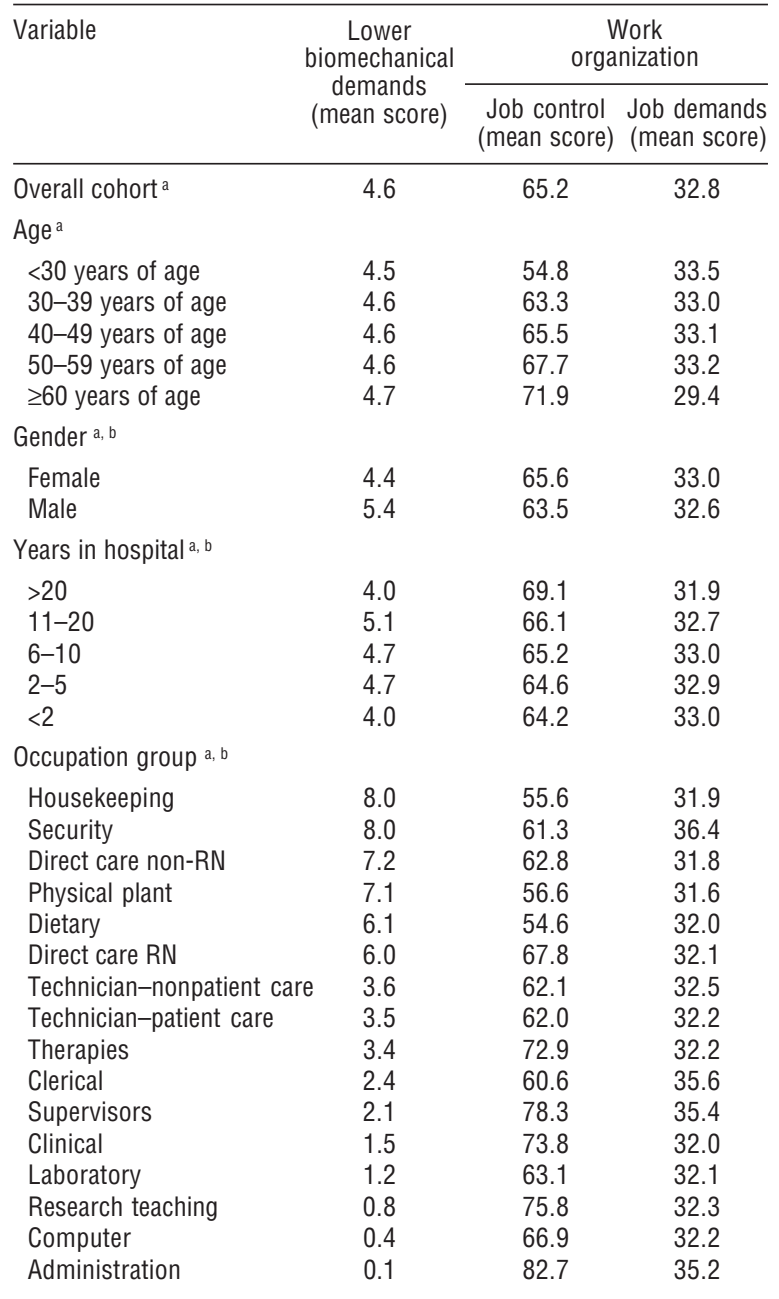

a Mean score: 0-14 for lower biomechanical demands, 47.8-89.5 for job control, and 28.4-36.9 for job demands.

b The work organization scores differed statistically across the demographic and job categories at the 0.05 level-analysis of variance.

graphic and biomechanical factors was taken into consideration. The current findings are consistent with those of other studies that have investigated the independent contribution of job control $(10,20)$ and objective workload measures on the risk for musculoskeletal injuries (8), including studies on workload measures (patient:nurse ratios) in the health care sector (11). Similar to the findings in this study, work by Kaila-Kangas and her colleagues (20) reported a threefold increased risk of hospitalization for back pain associated with low job control in a cohort of industrial employees in Finland, but no effect for psychological job demands was found.

While our study did not delineate the mechanisms by which work-organization factors are related to musculoskeletal injuries, other investigations among health 
Table 3. Injury rates and risk factors for lower-body musculoskeletal injuries (compensation claims) in a cohort of health care workers $(\mathrm{N}=3769)$. $(\mathrm{RR}=$ risk ratio, $95 \% \mathrm{Cl}=95 \%$ confidence interval)

\begin{tabular}{|c|c|c|c|c|c|}
\hline Study variable & $\begin{array}{l}\text { Rate } \\
\text { per100 } \\
\text { person- } \\
\text { years }\end{array}$ & $\begin{array}{c}\text { Uni- } \\
\text { variable } \\
\text { RR }\end{array}$ & $95 \% \mathrm{Cl}$ & $\begin{array}{l}\text { Multi- } \\
\text { variable } \\
\text { adjusted a } \\
\text { RR }\end{array}$ & $95 \% \mathrm{Cl}$ \\
\hline
\end{tabular}

\begin{tabular}{|c|c|c|c|c|c|}
\hline \multicolumn{6}{|l|}{ Demographic factors } \\
\hline \multicolumn{6}{|l|}{ Age } \\
\hline$<30$ years & 4.6 & 1.00 & . & 1.00 & . \\
\hline $30-39$ years & 4.1 & 0.90 & $0.70-1.16$ & 1.05 & $0.81-1.37$ \\
\hline 40-49 years & 4.2 & 0.92 & $0.70-1.20$ & 1.30 & $0.98-1.74$ \\
\hline$\geq 50$ years & 3.8 & 0.86 & $0.63-1.18$ & 1.40 & $0.95-2.06$ \\
\hline \multicolumn{6}{|l|}{ Gender } \\
\hline Female & 4.0 & 1.00 & . & 1.00 & . \\
\hline Male & 5.2 & 1.30 & $1.05-1.61$ & 1.06 & $0.84-1.32$ \\
\hline \multicolumn{6}{|l|}{ Years in job } \\
\hline$>10$ & 2.9 & 1.00 & . & 1.00 & . \\
\hline $6-10$ & 3.4 & 1.17 & $0.77-1.79$ & 1.22 & $0.79-1.88$ \\
\hline $2-5$ & 4.3 & 1.50 & $1.06-2.13$ & 1.62 & $1.12-2.34$ \\
\hline$<2$ & 4.7 & 1.64 & $1.17-2.31$ & 1.44 & $1.00-2.08$ \\
\hline \multicolumn{6}{|l|}{ Previous injury } \\
\hline $\begin{array}{l}\text { None/ } \\
>24 \text { months ago }\end{array}$ & 2.3 & 1.00 & . & 1.00 & . \\
\hline $\begin{array}{l}\text { Within past } \\
13-24 \text { months }\end{array}$ & 4.1 & 1.82 & $1.39-2.39$ & 1.71 & $1.30-2.25$ \\
\hline $\begin{array}{l}\text { Within past } \\
7-12 \text { months }\end{array}$ & 5.3 & 2.37 & $1.79-3.14$ & 2.13 & $1.60-2.84$ \\
\hline $\begin{array}{l}\text { Within past } \\
3-6 \text { months }\end{array}$ & 6.7 & 2.97 & $2.16-4.09$ & 2.59 & $1.87-3.58$ \\
\hline $\begin{array}{l}\text { Within past } \\
<3 \text { months }\end{array}$ & 10.0 & 4.42 & $3.15-6.20$ & 3.51 & $2.48-4.96$ \\
\hline \multicolumn{6}{|c|}{ Biomechanical demands } \\
\hline Low & 1.2 & 1.00 & . & 1.00 & . \\
\hline Medium & 3.7 & 2.59 & $1.77-3.80$ & 2.12 & $1.42-3.16$ \\
\hline High & 6.6 & 5.39 & $3.80-7.65$ & 3.36 & $2.27-4.98$ \\
\hline Very high & 7.4 & 6.05 & $4.02-9.09$ & 3.86 & $2.46-6.05$ \\
\hline \multicolumn{6}{|c|}{ Work organization factors } \\
\hline \multicolumn{6}{|l|}{ Job control b } \\
\hline High & 1.6 & 1.00 & . & 1.00 & . \\
\hline Medium-high & 4.1 & 2.63 & $1.74-3.97$ & 1.25 & $0.80-1.95$ \\
\hline Medium-low & 4.9 & 3.23 & $2.19-4.74$ & 1.64 & $1.08-2.49$ \\
\hline Low & 5.0 & 3.24 & $2.18-4.82$ & 1.58 & $1.02-2.44$ \\
\hline \multicolumn{6}{|l|}{ Job demands ${ }^{b}$} \\
\hline Low & 4.0 & 1.00 & . & 1.00 & . \\
\hline Medium & 4.8 & 1.18 & $0.77-1.80$ & 1.09 & $0.64-1.84$ \\
\hline High & 1.9 & 0.47 & $0.28-0.79$ & 0.86 & $0.46-1.61$ \\
\hline \multicolumn{6}{|c|}{ Exploratory analysis of workload measures } \\
\hline \multicolumn{6}{|c|}{ Dept absenteeism ${ }^{c}$} \\
\hline Low & 1.9 & 1.00 & . & 1.00 & . \\
\hline Medium-low & 3.2 & 1.89 & $1.26-2.85$ & 1.27 & $0.90-1.79$ \\
\hline Medium-high & 5.0 & 2.34 & $1.59-3.42$ & 1.71 & $1.25-2.33$ \\
\hline High & 6.7 & 2.97 & $2.01-4.39$ & 2.19 & $1.61-2.98$ \\
\hline \multicolumn{6}{|c|}{ Department overtime ${ }^{c}$} \\
\hline Low & 3.1 & 1.00 & . & 1.00 & . \\
\hline Medium-low & 4.9 & 1.54 & $1.18-2.00$ & 1.20 & $0.91-1.55$ \\
\hline Medium-high & 4.7 & 1.48 & $1.12-1.95$ & 1.21 & $0.88-1.55$ \\
\hline High & 4.1 & 1.28 & $0.97-1.69$ & 1.15 & $0.84-1.48$ \\
\hline
\end{tabular}

a Adjusted for all other variables.

${ }^{b}$ Categorized into quartiles and tertiles on the basis of the overall distribution in the study population.

c Absenteeism and overtime were calculated as the proportion of total paid hours within each department of the hospital by month and categorized into quartiles. care personnel indicate a relationship between measures of job control and psychological demands with stress reactions such as elevations in blood pressure (21) and elevated cortisol levels (22). Several physiological, psychological, and biomechanical mechanisms have been proposed as links between job strain due to adverse work-organization factors and musculoskeletal morbidity. The direct physiological effect of job strain may affect muscle tension with ensuing stress-related endocrine effects on musculoskeletal function (22). The psychological response of workers to job strain may also result in altered work behavior or work methods in a way that increases biomechanical strain that results in musculoskeletal injury (22). Workers who are mentally stressed, for example, may use more force to complete a patient lift or push a laundry cart than if they were not stressed. Others have proposed that altered psychological states due to job strain increase musculoskeletal symptoms by increasing pain perception (23) or that more severe pathological change may occur as a result of suppressing pain to meet job demands (24). Alternatively, job-strain factors such as the level of control over worktasks may define the magnitude and duration of exposure to biomechanical factors in a way that results in musculoskeletal injury. Laboratory-based work by Davis and his colleagues (25) on the effect of mental processing at work (decision making and pacing) concluded that simultaneous decision making during the performance of lifting tasks and, in combination with increased pacing, intensified measures of spinal loading. They hypothesized that mental stress resulted in less controlled biomechanical movements.

Our findings for psychological job demands suggested an inverse relationship with the risk of musculoskeletal injuries. Similar findings have been reported in the cardiovascular literature (26), as well as in the musculoskeletal literature (20), including a study among health care workers (12). Other studies have found a positive association between musculoskeletal injuries and high levels of psychological job demands after adjustment for biomechanical factors (8), including among health care workers (27). This sample of health care workers demonstrated very little variability in psychological job demand scores, even though the breadth of occupations was greater than that included in previous health care studies. The job-demand subscale has been described as less robust psychometrically (23).

Working during periods of high departmental levels of absenteeism, but not overtime, was found to be associated with an increased risk of musculoskeletal injuries. The investigation of workload measures was an exploratory analysis, and the association with departmental levels of absenteeism should be interpreted with caution as reverse causality (ie, musculoskeletal symptoms resulting in absenteeism) cannot be fully ruled out 
as a bias in our study. Although absenteeism hours directly related to workers' compensation disability claims were excluded from the measure constructed for this study, some musculoskeletal symptoms or minor injuries may result in short-term time away from work that could not be removed from the absenteeism hours measure (recorded as general sick time in the human resources data).

Although exploratory in nature, the results for workload measures warrant further discussion to help guide future studies. Higher departmental absenteeism translates into an increased number of worktasks to be completed by workers who must compensate for absent coworkers during their regular shift even when temporary replacement workers are used. As described by health care workers themselves to the investigators, temporary employees generally represent experienced workers within the health care setting and their occupation, but not experienced within the current compliment of patients on the shift or the unit of the hospital resulting in increased work to compensate for the temporary worker. It also represents an unplanned increase in workload. An unplanned surge in workload may be an important element of work organization associated with an elevated risk of musculoskeletal injuries (28). The lack of a significant association with overtime is understandable given the hypothesized importance of a surge or unexpected increase in workload. Overtime translates into more work due to a longer workday, not necessarily an increased rate of work or an unexpected surge in workload. The combination of an increase in the rate of work and the unanticipated surge in workload may be important factors in the casual pathway of workload and musculoskeletal morbidity.

Our findings suggest that the frequency, duration, and magnitude of biomechanical forces such as awkward postures, forces, repetition, patient lifting, and heavy lifting are related to an increased risk of musculoskeletal injuries among health care workers. Studies in the health care sector have documented two- to eightfold increased risks for musculoskeletal injuries with biomechanical factors $(12,29)$. This study has also confirmed findings from previous studies that prior musculoskeletal injuries are one of the most reliable predictive factors for subsequent injury (20), including injuries among health care workers (30).

This study was designed to address several methodological problems in the field of work organization and musculoskeletal injuries. First, the investigation of work-organization factors was based on multivariable analyses that adjusted for the potential confounding effects of demographic and biomechanical factors. Second, a job-exposure matrix for work-organization measures was developed from survey and administrative data to avoid sole reliance on self-reported measures. Self- reported estimates for work-organization factors may reflect individual differences in the perception of the job rather than actual differences in job characteristics (31). According to the job-exposure matrix of measures summed across persons as in our study, it seems more likely that the estimates reflect differences in job characteristics. Predicting job control and psychological demand scores from three random samples of workers over a 4-year time span helped to minimize the effect of misclassification due to individual differences and decrease the importance of individual biasing factors across occupational groups. Third, biomechanical factors associated with occupations were assessed by direct observation, and a major limitation of previous studies that have relied on self-reports of physical demands or job titles as surrogate measures was thereby overcome (32, 33). Fourth, the longitudinal design allowed for more than single point estimates of exposures.

There were several study limitations that should be acknowledged. The imputation techniques used to develop the job-exposure matrix may still result in misclassification when within-occupation differences are ignored. The current imputation technique used to calculate measures of job control and demand from survey data included demographic and occupational characteristics to account for some of the individual differences within an occupation.

Another study limitation was that observations of biomechanical factors may be less precise than direct measurements. Burdorf (32) found that between-occupation variance accounted for more of the total variance in biomechanical postures than the within-worker or between-worker variance. Given the importance of assessing exposure to multiple biomechanical factors in a large epidemiologic study, the use of direct observations and a composite score in our study provided a practical and feasible way to compare many jobs and avoid confounding by strongly correlated biomechanical exposures (34).

Reliance on compensated musculoskeletal injuries may exclude some workers who have musculoskeletal symptoms related to work but do not currently require medical attention or do not take time away from work due to disability, and some workers who reported their injury to the compensation system but did not receive time-loss benefits (eg, medical aid only or rejected claim). We re-ran our analysis for all musculoskeletal injury reports to the OHS department regardless of compensation status and found the same results for workorganization and biomechanical factors, although the effect size was greater for the compensated injury outcome.

Residual confounding by unmeasured individuallevel variables is a critique of multivariable models. For example, we did not have a measure of socioeconomic 
status in our models. In a highly unionized environment, the difference between socioeconomic statuses (as measured by income) is minimized among health care workers when they are compared with the general population. Income levels are highly correlated with occupational groups, but we were unable to include occupational groupings in our models because of high correlations with biomechanical measures. Given minimal variability in socioeconomic status and the lack of strong evidence to include socioeconomic indicators in an occupational cohort within one sector, we opted to investigate the effect of biomechanical demands over including occupational groupings as a surrogate for socioeconomic status. Finally, the possibility of bias associated with the observed relationship between musculoskeletal injury and the absenteeism measure in the current study warrants caution when the findings and further investigation of the measurement of workload in future studies are interpreted.

Workforce shortages among health care workers are an ongoing concern in the Canadian health care system, attributable to several factors, including an aging workforce and work environment conditions. Findings on the relative importance of risk factors for musculoskeletal injuries among health care workers are of increasing importance with respect to the allocation of limited resources, the setting of priorities, the development of workplace prevention strategies for modifiable work conditions, and the retention of an experienced workforce. Findings from this study indicate that a combination of interventions aimed at reducing the biomechanical demands associated with health care occupations, improving work-organization conditions, and providing modified return-to-work options for workers with prior musculoskeletal injuries are warranted.

Future studies can build upon the our findings by investigating the cumulative effect of work-organization exposures on injuries, ascertaining musculoskeletal outcomes from data sources other than self-reports or compensation systems, linking with more-detailed medical data on the clinical course of musculoskeletal episodes, investigating the mechanisms by which work-organization factors influence musculoskeletal morbidity, and improving upon the workload measures.

\section{Acknowledgments}

This research was supported in part by Health Canada through a National Health Research and Development Program (NHRDP) Research Training Award. The principal investigator received support during the writing of the paper from the Mustard Fellowship in Work Environment and Health, Institute for Work \& Health. The authors thank the employees and administration of $\mathrm{St}$ Paul's Hospital for their participation in the study.

\section{References}

1. Workers' Compensation Board of British Columbia. Health Care Industry: statistics 2003. Richmond (British Columbia); Workers' Compensation Board of British Columbia; 2004.

2. Bongers PM, de Winter CR, Kompier MAJ, Hildebrandt VH. Psychosocial factors at work and musculoskeletal disease [review]. Scand J Work Environ Health. 1993;19:297-312.

3. Hoogendoorn WE, van Poppel MNM, Bongers PM, Koes BW, Bouter LM. Systematic review of psychosocial factors at work and private life as risk factors for back pain. Spine. 2000;25:2114-25.

4. Karasek R, Theorell T. Healthy work: stress, productivity and the reconstruction of working life. Washington (DC): Basic Books; 1990.

5. Karasek RA. Job demands, job decision latitude and mental strain: implications for job redesign. Adm Sci Q. 1979; 24:285-307.

6. Amick BC III, Kawachi I, Coakley EH, Lerner D, Levine S, Colditz GA. Relationship of job strain and iso-strain to health status in a cohort of women in the United States. Scand J Work Environ Health. 1998;24:54-61.

7. Cheng Y, Kawachi I, Coakley, Schwartz J, Colditz G. Association between psychosocial work characteristics and health functioning in American women: prospective study. BMJ. 2000;320:1432-36.

8. Krause N, Ragland DR, Fisher JM, Syme SL. Psychosocial job factors, physical workload and incidence of work-related spinal injury: a 5 year prospective study of urban transit operators. Spine. 1998;23:2507-16.

9. Schibye B, Skov T, Ekner D, Christiansen JU, Sjøgaard G. Musculoskeletal symptoms among sewing machine operators. Scand J Work Environ Health. 1995;21:427-34.

10. Kivimäki M, Vahtera J, Ferrie JE, Hemingway H, Pentti J. Organisational downsizing and musculoskeletal problems in employees: a prospective study. Occup Environ Med. 2001;58:811-7.

11. Larese F, Fiorito A. Musculoskeletal disorders in hospital nurses: a comparison between two hospitals. Ergonomics. 1994;37:1205-11.

12. Josephson M, Lagerström M, Hagberg M, Wigaeus Hjelm E. Musculoskeletal symptoms and job strain among nursing personnel: a study over a three year period. Occup Environ Med. 1997;54:681-5.

13. Niedhammer I, Lert F, Marne M. Back pain and associated factors in French nurses. Int Arch Occup Environ Health. 1994;66:349-57.

14. Karasek R, Brisson C, Kawakami N, Houtman I, Bongers P, Amick B. The Job Content Questionnaire (JCQ): an instrument for internationally comparative assessments of psychosocial job characteristics. J Occup Health Psychol. 1998;3:322-55.

15. Johnson JV, Stewart WF. Measuring work organization exposure over the life course with a job-exposure matrix. Scand J Work Environ Health. 1993;19:21-8.

16. Statistics for the Applied Sciences. SAS procedures manual. Cary (NC): SAS Institute Inc; 2002.

17. Occupational Health and Safety Administration (OHSA). De- 
scription of OSHA's Workplace Risk Factor Checklist (DRAFT). Washington (DC): US Department of Labor; 1995.

18. Statistics and Epidemiology Research Corporation. EGRET Statistical Software. Seattle (WA): Statistics and Epidemiology Research Corporation; 1997.

19. Moulton LH, Dibley MJ. Multivariate time-to-event models for studies of recurrent childhood diseases. Int J Epidemiol. 1997;26:1334-9.

20. Kaila-Kangas L, Kivimäki M, Riihimäki H, Luukkonen R, Kirjonen J, Leino-Arjas P. Psychosocial factors at work as predictors of hospitalization for back injuries. Spine. 2004;29:1823-30

21. Fox M, Dwyer DJ, Ganster DC. Effects of stressful job demands and control on physiological and attitudinal outcomes in a hospital setting. Acad Manage J. 1993;36:289-318.

22. Hagberg M, Silverstein B, Wells R, Smith MJ, Hendrick HW, Carayon P, et al. Work Related Musculoskeletal Disorders (WMSDs): a reference book for prevention. London: Taylor \& Francis; 1995.

23. Sauter SL, Swanson NG. An ecological model of musculoskeletal disorders in office work. London: Taylor \& Francis; 1996.

24. Theorell T, Nordemar R, Michélsen H, Stockholm Music I Study Group. Pain thresholds during standardized psychological stress in relation to perceived psychosocial work situation. J Psychosomatic R. 1993; 37:299-305.

25. Davis KG, Marras WS, Heaney CA, Waters TR, Gupta P. The impact of mental processing and pacing on spine loading. Spine. 2002;23:2645-53.

26. Theorell T, Tsutsumi A, Hallquist J, Reuterwall C, Higstedt $\mathrm{C}$, Fredlund $\mathrm{P}$, et al. Decision latitude, job strain and myocardial infarction: a study of working men in Stockholm. Am J
Public Health. 1998;88:382-8.

27. Engels JA, van der Gulden JW, Senden TF, van't Hof B. Work related risk factors for musculoskeletal complaints in the nursing profession: results of a questionnaire survey. Occup Environ Med. 1996;53:636-41.

28. Engels JA, van der Gulden JWJ, Senden TF, Hertog CAWM, Kolk JJ, Binkhorst RA. Musculoskeletal complaints and physical workload among the nursing staff in nursing-homes. J Occup Med. 1994;36:338-46.

29. Johansson JA. Psychosocial work factors, physical workload and associated musculoskeletal symptoms among home care workers. Scand J Psychol. 1995;36:113-29.

30. Fuortes LJ, Shi Y, Zhang M, Zwerling C, Schootman M. Epidemiology of back injury in university hospital nurses from review of workers' compensation records and a casecontrol survey. J Occup Med. 1994;36:1022-6.

31. Smedley J, Egger P, Cooper C, Coggon D. Prospective cohort study of predictors of incident low back pain in nurses. BMJ. 1997;314:1225-8.

32. Burdorf A. Exposure assessment of risk factors for disorders of the back in occupational epidemiology [review]. Scand J Work Environ Health. 1992;18:1-9.

33. Kilbom A. Assessment of physical exposure in relation to work-related musculoskeletal disorders-what information can be obtained from systematic observations? Scand J Work Environ Health. 1994;20 special issue:30-45.

34. Punnett L. Ergonomic stressors and upper extremity disorders in vehicle manufacturing: cross sectional exposure-response trends. Occup Environ Med. 1998;55:414-20.

Received for publication: 20 April 2005 